\title{
Universal Pointsets for 2-Coloured Trees ${ }^{\star}$
}

\author{
Mereke van Garderen ${ }^{1}$, Giuseppe Liotta ${ }^{2}$, and Henk Meijer ${ }^{1}$ \\ 1 Roosevelt Academy, The Netherlands \\ $\{m$. vangarderen, h.meijer $\}$ @roac.nl \\ 2 Università di Perugia, Italy \\ liotta@iei.unipg.it
}

\begin{abstract}
Let $R$ and $B$ be two sets of distinct points such that the points of $R$ are coloured red and the points of $B$ are coloured blue. Let $\mathcal{G}$ be a family of planar graphs such that for each graph in the family $|R|$ vertices are red and $|B|$ vertices are blue. The set $R \cup B$ is a universal pointset for $\mathcal{G}$ if every graph $G \in \mathcal{G}$ has a straight-line planar drawing such that the blue vertices of $G$ are mapped to the points of $B$ and the red vertices of $G$ are mapped to the points of $R$. In this paper we describe universal pointsets for meaningful classes of 2-coloured trees and show applications of these results to the coloured simultaneous geometric embeddability problem.
\end{abstract}

\section{Introduction}

Let $G$ be a planar graph with $n$ vertices whose vertex set is partitioned into subsets $V_{0}, \ldots, V_{k-1}$ for some positive integer $1 \leq k \leq n$ and let $S$ be a set of $n$ distinct points in the plane partitioned into subsets $S_{0}, \ldots, S_{k-1}$ with $\left|S_{i}\right|=\left|V_{i}\right|(0 \leq i \leq k-1)$. We say that each index $i$ is a colour, $G$ is a $k$-coloured planar graph, and $S$ is a $k$ coloured set of points compatible with $G$. A $k$-coloured point-set embedding of $G$ on $S$ is a polyline drawing of $G$ such that each vertex of $V_{i}$ is mapped to a distinct point of $S_{i}$ and no two edges cross.

Let $\mathcal{G}$ be a family of $k$-coloured graphs such that for each colour all graphs in $\mathcal{G}$ have the same number of vertices of that colour, and let $S$ be a $k$-coloured set of points compatible with each $G \in \mathcal{G}$. Set $S$ is an $h$-bend universal pointset for the family $\mathcal{G}$ if each $G \in \mathcal{G}$ has a $k$-coloured point-set embedding on $S$ such that every edge of $G$ is drawn as a polyline having at most $h$ bends. $h$-Bend universal pointsets are the subject of extensive research in the graph drawing and combinatorial geometry literatures.

Pach and Wenger [17] consider the family of $n$-coloured planar graphs and prove that any pointset in general position is $O(n)$-bend universal for this family. In the same paper, the authors show that for some $n$-coloured planar graphs and for some $n$-coloured compatible sets of points $\Omega(n)$ bends per edge may be necessary. Kaufmann and Wiese [16] prove that every set of $n$ distinct points in the plane is 2-bend universal for all (1-coloured) planar graphs. Everett et al. [6] show how to construct a set of $n$ distinct points that is 1-bend universal for all planar graphs. On the negative side, De Fraysseix, Pach, and Pollack [4] show that a 0-bend universal pointset does not

\footnotetext{
* Research supported in part by MIUR under project AlgoDEEP prot. 2008TFBWL4.
} 
exist for the family of planar graphs. Gritzman, Mohar, Pach, and Pollack [10] prove that every set of $n$ distinct points in the plane is 0-bend universal for the outerplanar graphs with $n$ vertices and that this is the largest subfamily of the planar graphs having this property.

Several results are also known for $h$-bend universal pointsets of $k$-coloured planar graphs with $1<k<n$ and $h \geq 1$. Di Giacomo et al. [9] show that every set of $n$ distinct points is 1-bend universal for the properly 2-coloured caterpillars, the properly 2 -coloured wreaths, the 2-coloured paths, and the 2 -coloured cycles. Di Giacomo et al. [7] also prove that every set of $n$ distinct points is 5 -bend universal for the 2-coloured outerplanar graphs, while Badent et al. [1] prove that not all sets of $n$ distinct points can be $h$-bend universal for 2-coloured non-outerplanar graphs for any fixed constant $h$.

This paper is devoted to the study of 0-bend universal pointsets for 2-coloured trees. Since we only consider pointsets that support straight-line drawings, we shall simply say universal pointset to mean a 0-bend universal pointset. Also, for consistency with existing literature, we name the two colours of our trees as red and blue. Although 2colored trees may appear as a somewhat restricted subfamily of the $k$-colored planar graphs, there is a rich literature concerning universal pointsets of these trees. While the interested reader is referred to the survey by Kaneko and Kano [14], we briefly recall here some of the most recent findings that are more closely related to our results.

Abellanas et al. [2] show that any 2-coloured pointset $S$ such that either the convex hull of $S$ consists of all red points and no blue points or $S$ is a linearly separable bipartition (i.e., there exists a line that separates all blue points from the red ones) is a universal set for the properly 2-coloured paths. Brandes et al. [3] extend this result and show that a linearly separable bipartition is universal for the (non necessarily properly) 2-coloured paths. Results by Ikebe et al. [11] and by Kaneko and Kano [12] show that every set of $n$ distinct points in general position is universal for the 2-colored forests of size $n$ and consisting of at most two trees each having exactly one red vertex. Follow-up papers by Kaneko and Kano [13]15] extend this last result to forests with more than two trees under the assumption that the forest consists of either star-trees or trees whose sizes differ from one another by at most one vertex. Di Giacomo et al. [8] also study forests such that every tree has exactly one red vertex and prove that any set of points where the red points are in convex position is universal for these graphs. Finally, Estrella-Balderrama, Fowler, and Kobourov [5] describe universal pointsets for different families of 2- and 3 -coloured trees, namely the 2-coloured spiders, the 3-coloured caterpillars, and the 3 -coloured radius-two stars.

This paper describes three new, fairly general families of 2-coloured trees for which universal pointsets exist. Our results are as follows.

- There exists a universal pointset for the properly 2-coloured trees whose leaves all have the same colour (Theorem1, Section 2).

- There exists a universal pointset for the properly 2-coloured full binary trees (Theorem 2] Section 2).

- There exists a universal pointset for the rooted 2-coloured trees where every vertex has at most one child having a colour different from its own (Theorem 3, Section 3).

Finally, an application of the our results to the $k$-coloured simultaneous geometric embeddability problem [3] is given in Section 4](Corollaries 1, 2] and 3). 


\section{Properly 2-Coloured Trees}

In this section we consider universal pointsets of properly 2-coloured trees, i.e. 2 -coloured trees in which no two vertices of the same colour are adjacent. Unless stated otherwise, the trees considered in this section are not rooted. Throughout the paper, the blue vertices will be depicted as black dots and the red vertices as white dots. We start by considering properly 2 -coloured trees where all leaves have a same colour.

Theorem 1. There exists a universal pointset for the properly 2-coloured trees in which all leaves have the same colour.

Proof. Let $\mathcal{T}$ be the set of properly 2-coloured trees such that each tree in the set has $n_{r}$ red vertices and $n_{b}$ blue vertices and such that all leaves have the same colour. Without loss of generality assume that the leaves are red, which implies $n_{r} \geq n_{b}$. If $n_{r}=n_{b}$ the tree is a path and the statement holds by the result of Abellanas et al. [2]. Hence we prove the theorem for the case that $n_{r}>n_{b}$.

We draw a "downwards pointing" circular arc $\rho$ and a horizontal line $\sigma$ below it so that each point on $\rho$ is visible from each point on $\sigma$ as illustrated in Figure 1 Let $S$ be a point set defined as follows. Place an alternating sequence of red and blue points on $\rho$ with $n_{b}$ red and $n_{b}$ blue points. We place $n_{r}-n_{b}$ red points on $\sigma$. We now prove that $S$ is universal for $\mathcal{T}$ by presenting an algorithm that, for any tree $T \in \mathcal{T}$, computes a 2coloured point set embedding of $T$ on $S$ with straight-line edges. For an illustration see Figure 1, where some edges have been purposely drawn as curves to make the picture more readable.

We start with decomposing $T$ in a set of paths. A total of $n_{r}-n_{b}$ red leaves will not be included in this decomposition. Since $T$ is properly 2 -coloured, any path in $T$ alternates between red and blue vertices. Choose an arbitrary path in $T$ from a leaf $v$ to a vertex $w$ adjacent to a leaf. Add the path from $v$ to $w$ to the decomposition. The path from $v$ to $w$ is called the first path of $T$. Mark the vertices in the first path and repeat the decomposition into paths as follows. For any marked vertex $v$ that has an unmarked neighbour $w$ that is not a leaf, choose a path starting at $w$ and ending at a leaf $u$. If $w$ is blue, add the path to the decomposition and mark all vertices in this path. If $w$ is red, remove $u$ from the path, add the shortened path to the decomposition and mark all vertices in this path. Notice that all paths start and end at vertices of different colours. We will draw all such paths on $\operatorname{arc} \rho$, and the remaining $n_{r}-n_{b}$ red leaves on $\sigma$.

Let $v_{0}, v_{1}, \ldots, v_{p-1}$ be the first path. Place $v_{0}$ on the left or right-most point of $\rho$, depending on which point has the correct colour. Place the remaining vertices of the first path on neighbouring points of $S$ on $\rho$. We will now consider $T$ as a rooted tree, rooted at an arbitrary vertex on this path. Let $w_{0}, w_{1}, \ldots, w_{k-1}$ be the vertices in $T$ that do not lie on the first path, that are neighbours of vertices in the first path, and that are not leaves. Each $w_{i}$ is an endpoint of one of the paths in the decomposition. Assume that we have numbered these vertices from one end of the path to the other, i.e. for all $i<j$, if $w_{i}$ and $w_{j}$ are neighbours of vertices $v_{a}$ and $v_{b}$ in the first path then $a \leq b$. Let $n_{i}$ be the number of vertices in the subtree rooted at $w_{i}$ for $0 \leq i<k$. Processing the unused points on $\rho$ starting at the unused point besides $v_{p-1}$ reserve the first $n_{k-1}$ points for the paths in the subtree of $w_{k-1}$, reserve the next $n_{k-2}$ points for the paths 

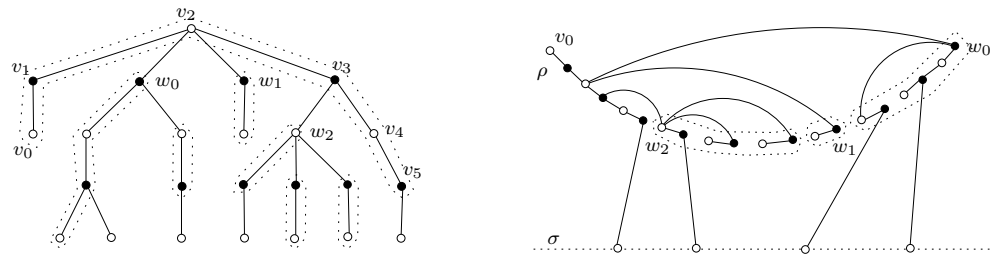

Fig. 1. Illustration for the proof of Theorem 1 The red points are white dots, the blue points are black dots. The first path has length 6 and $k=3, n_{0}=6, n_{1}=2$ and $n_{2}=6$. Some edges have been drawn as curves to make the figure more readable.

in the subtree of $w_{k-2}$, and so on. Then, for all $i$ place $w_{i}$ on the left or right most point of its reserved group of points, depending on which point has the correct colour. Map the remaining vertices on the path containing $w_{i}$ to the neighbouring points of $S$ on $\rho$. We then continue recursively. Finally, we can connect the points of $S$ on $\sigma$ to their neighbours on $\rho$, since each point on $\sigma$ can see all points on $\rho$. Since the resulting drawing is a 0 -bend point-set embedding of $T$ on $S$, it follows that $S$ is a universal pointset for $\mathcal{T}$.

Motivated by Theorem 1 it is natural to ask whether there exists a universal pointset also for properly 2 -coloured trees whose leaves can be either red or blue. The next theorem answers this question for the family of properly 2-coloured full binary trees, i.e. properly 2 -coloured trees whose vertices have degree either three or one. The proof is omitted here because of space limitations.

Theorem 2. There exists a universal pointset for the properly 2-coloured full binary trees.

\section{Almost Mono-Chromatic Rooted Trees}

In this section we study universal pointsets of 2-coloured trees where vertices of the same colour may be adjacent. The almost monochromatic trees are those 2-colored rooted trees where each parent has at most one child of a different colour than its own.

Theorem 3. There exists a universal pointset for the almost monochromatic trees.

Sketch of Proof: Let $\mathcal{T}$ be the set of almost monochromatic trees having $n_{r}$ red vertices and $n_{b}$ blue vertices. Let $S$ be a set of points defined as follows. Draw two circular $\operatorname{arcs} c_{r}$ and $c_{b}$ so that they form an "hourglas", as illustrated in Figure 2. Place $n_{r}$ red points on $c_{r}$ and $n_{b}$ red points on $c_{b}$. Assume without loss of generality that the root $v_{0}$ is red. We prove that $S$ is universal for $\mathcal{T}$ by showing how to compute a 0 -bend point set embedding on $S$ of any tree $T \in \mathcal{T}$. Call unfinished a vertex of $T$ whose children are not all drawn. We compute a 0-bend point set embedding of $T$ on $S$ by maintaining the following invariant: For each unfinished vertex $v \in T$ there exists a convex region that intersects $c_{r}$ and/or $c_{b}$ and such that $v$ is either mapped to the lowest red point or to the lowest blue point on $c_{r}$ or $c_{b}$ inside this region. The region associated with $v$ 
contains the correct number of vertices for the subtree of $v$. Map $v_{0}$ to the bottom red point on $c_{r}$. Notice that the invariant holds after the first step. If $v_{0}$ has a blue child $v_{b}$, assign a convex region to $v_{b}$ containing the unused bottom-most points from $c_{r}$ and $c_{b}$. The number of red and blue points in this region is equal to the number of red and blue vertices in the subtree rooted at $v_{b}$. Place $v_{b}$ in its region at the bottom unused point of $c_{b}$. If $v_{0}$ has red children, then for each red child $v_{r}$, assign a convex region to $v_{r}$ containing the next group of bottom-most points from $c_{r}$ and from $c_{b}$. Map the children of $v_{0}$ to the bottom most points on $c_{r}$ in their regions. Since the invariant holds for each child of $v_{0}$, each subtree can be recursively drawn inside the corresponding convex regions. Hence $S$ is a universal pointset for $\mathcal{T}$.
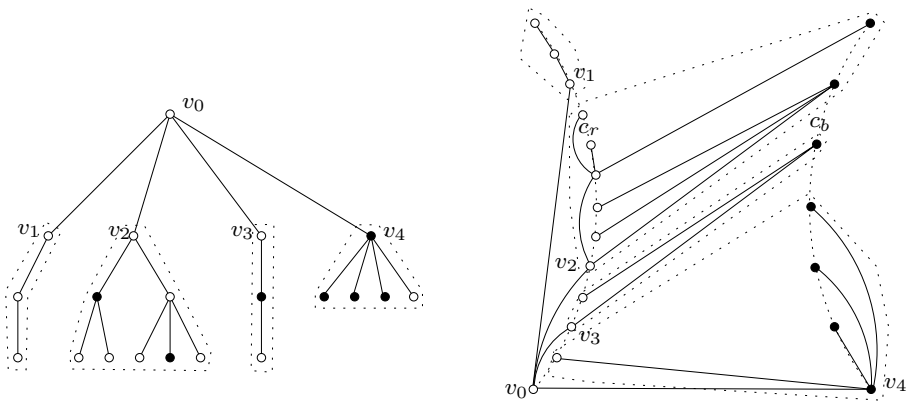

Fig. 2. Illustration for the proof of Theorem 3 The red points are white dots, the blue points are black dots. Some edges have been drawn as curves to make the figure more readable.

\section{Coloured Simultaneous Geometric Embeddings}

The $k$-coloured simultaneous geometric embeddability problem is defined as follows. The input is a set of $k$-coloured planar graphs $G_{1}=\left(V, E_{1}\right), G_{2}=\left(V, E_{2}\right), \ldots, G_{r}=$ $\left(V, E_{r}\right)$ on the same vertex set $V$. The goal is to find planar straight-line drawings $D_{i}$ of $G_{i}$ using the same $|V|$ points in the plane for all $i=1, \ldots, r$, such that vertices of colour $i$ are mapped to points of colour $i$. The set of drawings $D_{i}(i=1, \ldots, r)$ is a $k$-coloured simultaneous geometric embedding of the graphs $G_{1}=\left(V, E_{1}\right), G_{2}=\left(V, E_{2}\right), \ldots$, $G_{r}=\left(V, E_{r}\right)$. The $k$-coloured simultaneous geometric embeddability problem was first defined by Brandes et al. [3] and subsequently investigated by Estrella-Balderrama, Fowler, and Kobourov [5]. By using the universal pointsets of Theorems 2, 1 , and 3 the following results are immediate.

Corollary 1. Any number of properly 2-coloured full binary trees admits a 2-coloured simultaneous geometric embedding.

Corollary 2. Any number of properly 2-coloured trees whose leaves all have the same colour admits a 2-coloured simultaneous geometric embedding.

Corollary 3. Any number of rooted almost-monochromatic 2-coloured trees admits a 2-coloured simultaneous geometric embedding. 


\section{Open Problems}

It is unknown whether there are universal pointsets for properly 2-coloured (binary) trees, or more generally whether there are universal pointsets for 2-coloured trees.

\section{References}

1. Badent, M., Giacomo, E.D., Liotta, G.: Drawing colored graphs on colored points. Theor. Comput. Sci. 408(2-3), 129-142 (2008)

2. Abellanas, M., Garcia-Lopez, J., Hernández-Peñver, G., Noy, M., Ramos, P.A.: Bipartite embeddings of trees in the plane. Discrete Applied Mathematics 93(2-3), 141-148 (1999)

3. Brandes, U., Erten, C., Fowler, J.J., Frati, F., Geyer, M., Gutwenger, C., Hong, S.-H., Kaufmann, M., Kobourov, S.G., Liotta, G., Mutzel, P., Symvonis, A.: Colored simultaneous geometric embeddings. In: Lin, G. (ed.) COCOON 2007. LNCS, vol. 4598, pp. 254-263. Springer, Heidelberg (2007)

4. de Fraysseix, H., Pach, J., Pollack, R.: How to draw a planar graph on a grid. Combinatorica 10(1), 41-51 (1990)

5. Estrella-Balderrama, A., Fowler, J.J., Kobourov, S.G.: Colored simultaneous geometric embeddings and universal pointsets. In: Proceedings of the 21st Canadian Conference on Computational Geometry (CCCG 2009), pp. 17-20 (2009)

6. Everett, H., Lazard, S., Liotta, G., Wismath, S.K.: Universal sets of points for onebend drawings of planar graphs with vertices. Discrete \& Computational Geometry 43(2), 272-288 (2010)

7. Giacomo, E.D., Didimo, W., Liotta, G., Meijer, H., Trotta, F., Wismath, S.K.: k-colored pointset embeddability of outerplanar graphs. J. Graph Algorithms Appl. 12(1), 29-49 (2008)

8. Giacomo, E.D., Didimo, W., Liotta, G., Meijer, H., Wismath, S.K.: Point-set embeddings of trees with given partial drawings. Comput. Geom. 42(6-7), 664-676 (2009)

9. Giacomo, E.D., Liotta, G., Trotta, F.: On embedding a graph on two sets of points. Int. J. Found. Comput. Sci. 17(5), 1071-1094 (2006)

10. Gritzmann, P., Mohar, B., Pach, J., Pollack, R.: Embedding a planar triangulation with vertices at specified points. Amer. Math. Monthly 98(2), 165-166 (1991)

11. Ikebe, Y., Perles, M.A., Tamura, A., Tokunaga, S.: The rooted tree embedding problem into points in the plane. Discrete \& Computational Geometry 11, 51-63 (1994)

12. Kaneko, A., Kano, M.: Straight-line embeddings of two rooted trees in the plane. Discrete \& Computational Geometry 21(4), 603-613 (1999)

13. Kaneko, A., Kano, M.: Straight line embeddings of rooted star forests in the plane. Discrete Applied Mathematics 101(1-3), 167-175 (2000)

14. Kanenko, A., Kano, M.: Discrete geometry on red and blue points in the plane - a survey. In: Discrete and Computational Geometry. Algorithms and Combinatories, vol. 25, pp. 551-570. Springer, Heidelberg (2003)

15. Kaneko, A., Kano, M.: Semi-balanced partitions of two sets of points and embeddings of rooted forests. Int. J. Comput. Geometry Appl. 15(3), 229-238 (2005)

16. Kaufmann, M., Wiese, R.: Embedding vertices at points: Few bends suffice for planar graphs. Journal of Graph Algorithms and Applications 6(1), 115-129 (2002)

17. Pach, J., Wenger, R.: Embedding planar graphs at fixed vertex locations. Graph and Combinatorics $17,717-728$ (2001) 